\title{
DIGESTIBILIDAD Y ENERGÍA DIGESTIBLE DE CINCO LEGUMINOSAS FORRAJERAS TROPICALES
}

\author{
Alejandrina Sotelo M..$^{*}$, Carlos Contreras M. ${ }^{1}$, Edgar Norabuena M. ${ }^{2}$, \\ Roxana Castañeda S. ${ }^{3}$, Mariella van Heurck ${ }^{4}$, Luz Bullón C. ${ }^{5}$
}

\begin{abstract}
RESUMEN
El experimento fue conducido para determinar los coeficientes de digestibilidad y la energía digestible del Arachis pintoi, Stylozanthes guianensis, Erythrina poeppigiana, Centrocema macrocarpum y Pueraria phaseoloides. Se empleo el método directo, usando el mismo forraje como único alimento. El experimento tuvo dos fases, una de adaptación y una de colección de heces, con una duración de 15 y 5 días, respectivamente. Se utilizaron 25 cuyes machos en etapa de crecimiento distribuidos de tal manera que 5 cuyes correspondieron a cada leguminosa tropical evaluada. Los coeficientes de digestibilidad de los nutrientes del Arachis pintoi $(51,96,56,80,29,46$ y 71,32 por ciento de digestibilidad de la materia seca, proteína cruda, fibra cruda y extracto libre de nitrógeno, respectivamente) fueron cuantitativamente superiores a los otros tratamientos a excepción de la ceniza. Asimismo, los valores de energía digestible (en base seca) obtenidos fueron mayores en el Arachis pintoi $(2,20 \mathrm{Kcal} / \mathrm{g})$ que los otros forrajes.
\end{abstract}

Palabras clave: Energía digestible, digestibilidad, leguminosa tropical, Arachis pintoi, Stylozanthes guianensis, Erythrina poeppigiana, Centrocema macrocarpum, Pueraria phaseoloides, cuyes.

\section{DIGESTIBILITY AND DIGESTIBLE ENERGY OF FIVE TROPICAL FORAGE TREE LEGUMES}

\begin{abstract}
This study was carried out to determine the digestibility coefficients and digestible energy of Arachis pintoi, Stylozanthes guianensis, Erythrina poeppigiana, Centrocema macrocarpum and Pueraria phaseoloides. The direct method was used, using the same forage as the only

\footnotetext{
${ }^{1}$ Facultad de Zootecnia, Universidad Nacional Agraria La Molina, Lima, Perú. Apdo. Postal 12-01-056 LimaPerú. asotelo@lamolina.edu.pe

${ }^{2}$ Facultad de Química, Universidad Nacional de Ingeniería, Lima, Perú

${ }^{3}$ Facultad de Ciencias Veterinarias y Biológicas, Universidad Científica del Sur, Lima, Perú.

${ }^{4}$ Facultad de Zootecnia, Universidad Nacional de la Amazonia Peruana, Lima, Perú.

${ }^{5}$ Facultad de Economía y Planificación. Departamento de Estadística. Universidad Nacional Agraria La Molina, Lima, Perú.
} 
food. The experiment had two phases, one for adaptation and one of faeces collection, with duration of 15 and 5 days, respectively. We used 25 male guinea pigs in growth stage distributed in order to get 5 guinea pigs for each tropical legume evaluated. The digestibility coefficients of nutrients were numerically higher for Arachis pintoi $(51,96,56,80,29,46$ y 71,32 percent of the dry matter, crude protein, crude fiber and nitrogen free extract digestibility, respectively) than for all the other treatments, except for the ash. Likewise, digestible energy values (in dry basis) were greater for Arachis pintoi $(2,20 \mathrm{kcal} / \mathrm{g})$ than for all other forages.

Key words: Digestible energy, digestibility, tropical legumes, Arachis pintoi, Stylozanthes guianensis, Erythrina poeppigiana, Centrocema macrocarpum, Pueraria phaseoloides, guinea pig.

\section{INTRODUCCIÓN}

El cuy es un roedor que pertenece al género Cavia, tiene su origen en los andes interandinos de América del Sur, siendo el Cavia cutleri el antecesor de la especie doméstica actualmente conocida.

El cuy es una especie de alta adaptación a los diferentes medios ecológicos por lo que es posible encontrarlo en zonas de selva. La disponibilidad de alimentos de elevado valor nutricional para la producción animal es cada vez mayor, por lo que se hace necesario disponer de insumos de bajo costo y, sobre todo, que no compitan con los insumos usados en la alimentación de monogástricos, incluyendo el hombre. Los forrajes son la base de la alimentación en los cuyes, siendo potencialmente usados como insumo en la alimentación animal; por ello, es importante identificar los pastos en nuestra selva peruana, sobre todo las leguminosas de gran nivel proteico. Por esta razón, la Asamblea General de las Naciones Unidas consideró denominar el año 2016 como el "Año Internacional de las Legumbres" $\mathrm{FAO}^{14}$. En este contexto, resulta valioso el poder registrar los conocimientos asociados al valor nutricional de las leguminosas tropicales, con énfasis en la alimentación de cuyes.

El III Censo Nacional Agropecuario, realizado en Perú en 1994, estimó que la selva aporta un 13 por ciento del total de cabezas de cuyes y 12,1 por ciento de unidades agropecuarias (UA) en el Perú. Los cuyes se crían en unidades animales para la venta en los mercados y en forma ambulatoria, autoconsumo, fiestas y ceremonias. De esta manera, la selva del Perú constituye una importante plaza para la crianza de cuyes, representando una excelente oportunidad para la introducción de insumos nuevos de bajo costo.

Una crianza rentable requiere manejo intensivo y disponibilidad de recursos forrajeros, por lo que se hace necesario buscar insumos alimenticios no tradicionales adaptables a las zonas de trópico y de bajo costo. Por esta razón y para este estudio se han escogido cinco leguminosas tropicales, las cuales, desde el punto de vista nutricional y económico, son potencialmente excelentes alternativas en la alimentación de los cuyes. En tal sentido, el estudio contribuirá con información nutricional de los mencionados insumos para la actualización de nuevas 
tablas de composición de alimentos y de esta manera puedan ser usados como insumos para alimentación de cuyes en la crianza comercial.

\section{PARTE EXPERIMENTAL}

\section{Leguminosas forrajeras evaluadas}

Se evaluaron cinco leguminosas forrajeras provenientes del distrito de Yurimaguas, en la Región Loreto de la Amazonía peruana. Los pastos fueron cortados como se indica:

- Centrosema macrocarpum.- A los 70 días.

- $\quad$ Pueraria phaseoloides.- A los $70 \mathrm{~cm}$ de altura (aproximadamente de 2,5 a 3 meses de edad).

- Stylosanthes guianensis.- Corte cuando los tallos alcanzaron $70 \mathrm{~cm}$ de altura.

- Erythrina poeppigiana.- Poda del árbol a los 6 meses desde el último corte.

- $\quad$ Arachis pintoi.- A los 60 días.

Las cinco leguminosas fueron secadas y luego molidas utilizando un molino de seis cuchillas y una malla de $2 \mathrm{~mm}$ para obtener harina de cada una. Posteriormente, se tomó una muestra de cada una de ellas, las cuales fueron sometidas al análisis químico proximal según la metodología de la $\mathrm{AOAC}^{1}$.

\section{Animales}

Se usaron 25 cuyes machos mejorados de tres meses de edad y peso homogéneo, los que fueron facilitados por la Granja de Cuyes de Cieneguilla del Programa de Investigación y Proyección Social en Carnes (PIPS-Carne), Facultad de Zootecnia de la Universidad Nacional Agraria La Molina.

\section{Variables evaluadas}

El experimento tuvo un periodo de adaptación de quince días de duración para cada ensayo, realizándose un cambio gradual del alimento (concentrado y el forraje de evaluación) en los primeros cuatro días y proporcionando sólo la leguminosa a evaluar en los últimos 11 días. La alimentación fue ad limitum, cuantificándose diariamente el suministro de forraje seco, para ello se pesó diariamente durante los 15 días, lo ofrecido y lo residual, a fin de calcular el consumo neto.

El periodo de colección de heces se realizó los últimos cinco días para cada uno de los grupos de leguminosas, utilizando el método directo de colección total de heces. Las heces se mezclaron homogéneamente al final del periodo de colección realizándose un composito por cada animal. Una parte fue llevada a la estufa $\left(105^{\circ} \mathrm{C}\right)$ por 24 horas para determinar la materia seca de las heces y otra parte presecada a $60{ }^{\circ} \mathrm{C}$ por 24 horas, luego molida y etiquetada para analizar posteriormente su composición química proximal. 
En base a los resultados obtenidos del análisis proximal de heces y forraje, se determinó el porcentaje de digestibilidad aparente de los nutrientes de las muestras de leguminosas, aplicando el método directo de digestibilidad descrito por Crampton y Harris ${ }^{2}$ :

$$
\mathrm{CD}(\%)=\frac{\text { Nutriente ingerido }- \text { Nutriente heces }}{\text { Nutriente ingerido }}
$$

Donde:

$\mathrm{CD}=$ Coeficiente de digestibilidad aparente

Para la determinación de la energía digestible de las leguminosas, previamente se estimó la energía bruta en base a los porcentajes de nutrientes de las cinco leguminosas y de sus respectivas heces, determinados por análisis químico proximal; luego se multiplicó por sus valores calóricos promedios (proteína: $5,7 \mathrm{kcal} / \mathrm{g}$; extracto etéreo: 9,3 Kcal/g; extracto libre de nitrógeno y fibra: 4,1 kcal/g) según la Alimentation Equilibree Commentry3. Finalmente, en base al contenido energético del alimento y las heces expresadas en base seca, se determinó la energía digestible del forraje, mediante la fórmula descrita por Crampton y Harris²:

$$
\mathrm{ED}=\mathrm{EB}-\frac{\mathrm{EH} \times \mathrm{Qh}}{\mathrm{Ia}}
$$

Donde:

$\mathrm{ED}=$ Energía digestible de la leguminosa $(\mathrm{kcal} / \mathrm{g})$

$\mathrm{EB}^{*}=$ Energía bruta de la leguminosa $(\mathrm{kcal} / \mathrm{g})$

$\mathrm{Qh}=$ Cantidad de heces producidas por día $(\mathrm{g})$

$\mathrm{EH}^{*}=$ Energía Bruta de las heces $(\mathrm{kcal} / \mathrm{g})$

$\mathrm{Ia}=$ Cantidad de alimento ingerido por día $(\mathrm{g})$

* La energía bruta de la leguminosa y de las heces se calculó con la siguiente fórmula descrita por Alimentation Equilibree Commentry ${ }^{3}$ :

$$
\mathrm{EB}=5,7 \times(\% \mathrm{PC})+9,3 \times(\% \mathrm{EE})+4,1 \times(\% \mathrm{FC}+\% \mathrm{ELN})
$$

\section{Análisis estadístico}

La agrupación de datos obtenidos de cada variable en este experimento fue resumida mediante un análisis descriptivo utilizando al promedio como medida de tendencia y la desviación estándar y el coeficiente de variabilidad como medidas de variabilidad.

\section{Consumo voluntario}

\section{RESULTADOS Y DISCUSIÓN}

Los consumos de los forrajes secos y molidos de las cinco leguminosas forrajeras varían entre 51 y $70 \mathrm{~g}$, como se observa en el cuadro 1 . 
Cuadro 1. Efecto del tipo de leguminosa sobre el consumo voluntario (g/cuy)

\begin{tabular}{cccccc}
\hline $\begin{array}{c}\text { Animal } \\
\mathbf{N}^{\circ}\end{array}$ & $\begin{array}{c}\text { Pueraria } \\
\text { phaseoloides }\end{array}$ & $\begin{array}{c}\text { Stylozanthes } \\
\text { guianensis }\end{array}$ & $\begin{array}{c}\text { Erythrina } \\
\text { poeppigiana }\end{array}$ & $\begin{array}{r}\text { Centrocema } \\
\text { macrocarpum }\end{array}$ & $\begin{array}{c}\text { Arachis } \\
\text { pintoi }\end{array}$ \\
\hline 1 & 70,20 & 68,80 & 71,70 & 70,60 & 46,30 \\
2 & 62,40 & 61,90 & 68,80 & 63,30 & 52,80 \\
3 & 72,20 & 51,20 & 67,80 & 64,80 & 47,10 \\
4 & 67,30 & 72,60 & 73,30 & 57,10 & 49,70 \\
5 & 58,10 & 65,20 & 68,40 & 55,00 & 57,00 \\
Promedio & $\mathbf{6 6 , 0 4}$ & $\mathbf{6 3 , 9 4}$ & $\mathbf{7 0 , 0 0}$ & $\mathbf{6 2 , 1 6}$ & $\mathbf{5 0 , 5 8}$ \\
\hline
\end{tabular}

Los forrajes con mayor contenido de fibra han obtenido los mayores consumos, posiblemente debido a que los animales trataron de compensar las deficiencias propias de estos pastos con un mayor consumo. Esto no se observa en el Arachis pintoi, que presenta el menor consumo voluntario, lo cual puede deberse al menor contenido de fibra cruda y mayor contenido de extracto libre de nitrógeno comparado a las otras leguminosas evaluadas. También puede deberse a su energía digestible, ya que esta fue superior a la de las demás leguminosas; y ello probablemente contribuyó a disminuir el consumo. Además, Waghorn et al. ${ }^{4}$ indicaron que el Arachis pintoi posee una frágil partícula debido en gran medida al menor contenido de pared celular, el cual permite proveer mayor cantidad de nutrientes. La interacción de nutrientes dentro del mismo forraje y el grado de madurez de la planta afectan en algún grado el consumo ${ }^{5}$ incrementándolo o reduciéndolo. Se debe considerar que dependiendo del grado de aceptación del alimento y principalmente de la composición nutritiva será el consumo que se produzca en cada una de las etapas fisiológicas de los animales, por lo tanto a mejor calidad, menor cantidad y viceversa ${ }^{6}$. En general, altos consumos y baja digestibilidad de un alimento pueden estar relacionados al mayor contenido de fibra, lo que produce una tasa rápida de pasaje a través del tracto digestivo, disminuyendo la absorción de los nutrientes. El consumo de alimento también se incrementa con la concentración de proteína cruda en la dieta. Por otro lado, los bajos consumos asociados con alta digestibilidad de un alimento pueden estar relacionados a la presencia de compuestos depresores del apetito, como taninos y alcaloides ${ }^{7}$. La leguminosa que presentó el menor consumo (Arachis pintoi), cuenta con taninos condensados que pueden variar con el periodo de corte. Por ejemplo, se pueden encontrar cantidades de taninos condensados de 74,1 g/Kg MS a corte de seis meses de establecidas y $85,8 \mathrm{~g} / \mathrm{Kg}$ MS a cortes efectuados a 28 días $^{8}$.

\section{Composición química}

Los valores obtenidos, tanto para la proteína bruta como para la fibra bruta en base seca de las leguminosas tropicales, se encuentran dentro de los porcentajes establecidos por Skerman', quien también indica que los valores de fibra bruta suelen aumentar al progresar la madurez. Las composiciones químicas en base seca de las cinco leguminosas tropicales se presentan en el cuadro 2. 
Cuadro 2. Análisis químico proximal en base seca de las cinco leguminosas tropicales

\begin{tabular}{lccccccc}
\hline Leguminosa tropical & MO, (\%) & MS, (\%) & PC, (\%) & EE, (\%) & FC, (\%) & ELN, (\%)C, (\%) \\
\hline $\begin{array}{lllllll}\text { Pueraria phaseoloides } \\
\text { Erythrina poeppigiana }\end{array}$ & 82,52 & 100,00 & 19,76 & 1,98 & 35,76 & 35,02 & 7,48 \\
$\begin{array}{l}\text { Stylozanthes guianensis } \\
\text { Centrocema }\end{array}$ & 93,82 & 100,00 & 24,81 & 4,36 & 29,38 & 29,05 & 12,40 \\
macrocarpum & 84,77 & 100,00 & 14,48 & 1,96 & 37,96 & 39,42 & 6,18 \\
Arachis pintoi & 89,16 & 100,00 & 18,59 & 1,55 & 23,18 & 45,84 & 10,85 \\
\hline
\end{tabular}

MS: Materia seca, PC: Proteína cruda, EE: Extracto etéreo, FC: Fibra cruda, ELN: Extracto libre de nitrógeno, C: Ceniza, MO: materia orgánica, BF: Base fresca.

Fuente: Análisis proximal, realizado en el Laboratorio de Evaluación Nutricional de Alimentos (LENA).

\section{Digestibilidad aparente}

Los coeficientes de digestibilidad aparente de los nutrientes de las cinco leguminosas se presentan en el cuadro 3 , donde se observa que cuantitativamente la mejor digestibilidad aparente para todos los nutrientes corresponde al Arachis pintoi. Este resultado es similar al obtenido por Lagunes ${ }^{8}$, quien determinó y comparó el valor nutricional de cuatro leguminosas tropicales: Stylosanthes guianensis, Centrosema macrocarpum, Pueraria phaseoloides y Arachis pintoi, obteniendo este último la mayor digestibilidad de materia seca (74,4\%). Esto podría ser el resultado del bajo contenido de fibra cruda del Arachis pintoi comparado a las otras leguminosas. El consumo de materia seca, el contenido de fibra cruda, y de proteína cruda de la dieta tienen efecto sobre la digestibilidad de los nutrientes ${ }^{10}$.

La digestibilidad es afectada por diversos factores, siendo la fibra cruda el principal componente que afecta negativamente la digestibilidad de un insumo o dieta. La edad de corte del pasto influye de manera notable pues a mayor edad del pasto, aumenta la pared celular y disminuye el contenido celular, volviéndose menos digestible, además el bajo contenido celular del forraje también puede afectar el aprovechamiento del mismo. El extracto libre de nitrógeno está constituido principalmente por los carbohidratos solubles, así como también vitaminas y demás compuestos orgánicos solubles no nitrogenados; los cuales están inversamente relacionados con la cantidad de fibra presente en los forrajes, dado que a medida que esta aumenta, los carbohidratos solubles irán en descenso e influirá en la digestibilidad de los forrajes ${ }^{11}$. Por otro lado, a medida que maduran las leguminosas tropicales suele producirse una reducción en el porcentaje de proteína cruda de la planta que va asociada con la disminución de la digestibilidad de la proteína ${ }^{9}$ y un creciente aumento del contenido de la pared celular con la madurez ${ }^{12}$, debido a que la proteína cruda y pared celular interactúan en forma inversa ${ }^{13}$. 
Cuadro 3. Coeficientes de digestibilidad aparente (\%) y energía digestible (kcal/g) de las cinco leguminosas estudiadas

\begin{tabular}{lcccccccc}
\hline $\begin{array}{l}\text { Leguminosas } \\
\text { MO }\end{array}$ & $\mathbf{M S}^{(1)}$ & $\mathbf{P C}^{(\mathbf{1})}$ & $\mathbf{E E}^{(\mathbf{1})}$ & $\mathbf{F C}^{(\mathbf{1})}$ & $\mathbf{E L N}^{(1)}$ & $\mathbf{C}^{(\mathbf{1})}$ & $\mathbf{E D}^{(2)}$ \\
\hline $\begin{array}{l}\text { Pueraria } \\
\text { phaseoloides }\end{array}$ & 36,97 & 35,36 & 53,94 & 14,46 & 17,84 & 48,21 & 15,38 & 1,58 \\
$\begin{array}{l}\text { Stylozanthes } \\
\text { guianensis }\end{array}$ & 37,60 & 37,91 & 43,86 & 8,33 & 18,50 & 55,15 & 42,63 & 1,56 \\
$\begin{array}{l}\text { Erytrhina } \\
\text { poeppigiana }\end{array}$ & 23,87 & 24,09 & 41,52 & 8,87 & 18,22 & 16,77 & 25,61 & 1,04 \\
$\begin{array}{l}\text { Centrocema } \\
\begin{array}{l}\text { macrocarpum } \\
\text { Arachis }\end{array}\end{array}$ & 36,06 & 32,27 & 49,08 & 4,87 & 26,44 & 45,02 & 11,16 & 1,38 \\
pintoi & 56,51 & 51,96 & 56,80 & 19,42 & 29,46 & 71,32 & 14,50 & 2,20 \\
\hline
\end{tabular}

MO: Materia orgánica, MS: Materia seca, PC: Proteína cruda; EE: Grasa total, FC: Fibra cruda, ELN: Extracto libre de nitrógeno, C: Ceniza, ED: Energía digestible

(1) Los coeficientes de digestibilidad (CD) se determinaron aplicando el método directo de digestibilidad descrito por Crampton y Harris ${ }^{2}$.

(2) La energía digestible del forraje en base seca se calcula mediante la fórmula descrita por Crampton y Harris ${ }^{2}$.

\section{Energía digestible}

El contenido de energía digestible, correspondiente a cada una de las leguminosas estudiadas, se consignan en el cuadro 3, donde se observa que la energía digestible del Arachis pintoi fue 2,20 Mcal/kg, valor superior al encontrado en las otras leguminosas: Pueraria phaseoloides, Stylozanthes guianensis, Centrosema macrocarpum y Erythrina poeppigiana (1,58, 1,56, 1,38 y $1,04 \mathrm{Mcal} / \mathrm{kg}$ de energía digestible, respectivamente). Los mayores valores de energía digestible del Arachis pintoi en comparación a los demás forrajes se explica por la menor cantidad de fibra cruda (23,18 por ciento) y mayor cantidad de ELN (44,97 por ciento) al ser comparada con las demás leguminosas (valores que van desde 29,38 a 40,49 por ciento para la fibra cruda y de 26,72 a 39,42 por ciento para el ELN), permitiendo que la energía sea aprovechada mejor, dado que el ELN contiene carbohidratos solubles de fácil digestión. Además, la fragilidad de la molécula de Arachis pintoi ${ }^{4}$, debido posiblemente al arreglo estructural de este forraje influyó en que el valor de la energía digestible se incremente.

\section{CONCLUSIONES}

El Arachis pintoi obtuvo cuantitativamente la mayor digestibilidad y energía digestible que las otras leguminosas forrajeras tropicales. 


\section{AGRADECIMIENTO}

Los autores agradecen al laboratorio de Evaluación Nutricional de Alimentos del Departamento Académico de Nutrición de la Facultad de Zootecnia de la Universidad Nacional Agraria La Molina por el apoyo con los análisis requeridos para el presente trabajo de investigación.

\section{REFERENCIAS BIBLIOGRÁFICAS}

1. AOAC. Official Methods of Analysis of the Association of Official Analytical Chemist. $16^{\text {th }}$ Ed. Washington DC: Ed. Cunniff; 2012.

2. Crampton EW, Harris LE. Nutrición Animal Aplicada: El uso de los alimentos en la formulación de raciones para el ganado. $2^{\mathrm{da}}$ ed. Zaragoza: Editorial Acribia; 1974.

3. Alimentation Equilibree Commentry (AEC). Animal Feeding: Energy, Amino Acids, Vitamins, Minerals. Paris: Doc. No. 4. AEC, Commentry; 1978.

4. Waghorn G, Shelton I, Thomas V. Particle breakdown and rumen digestion of fresh ryegrass (Lolium perenne $L$.) and lucerne (Medicago sativa $L$.) fed to cows during a restricted feeding period. Br J Nutr. 1988; 61(3): 409-423.

5. Rommey DL, Gill M. Intake of Forages. In: Givens DI, Owen E, Axford RFE, Omed HM. Forage Evaluation in Ruminant Nutrition. Wallingford: CABI Publishing; 2000. p. 43-62.

6. Meza G, Cabrera R, Morán J, Meza F, Cabrera C, Meza C, Meza J, Cabanilla M, López F, Pincay J, Bohórquez T, Ortiz J. Mejora de engorde de cuyes (Cavia porcellus L.) a base de gramíneas y forrajeras arbustivas tropicales en la zona de Quevedo, Ecuador. IDESIA (Chile). 2014; 32(3): 75-80.

7. Tshibangu I, Nsahlai V, Kiatoko H, Hornick JL. Nutritive value of Adenodolichos rhomboideus leaves compared with Leucaena leucocephala and Stylosanthes guianensis forages in indigenous goats in Lubumbashi (DR Congo). Biotechnol Agron Soc Environ. 2014; 18(2): 165-173.

8. Lagunes S. Evaluación productiva y de calidad de leguminosas tropicales en el estado de Puebla [Tesis para optar el título de Maestro Tecnólogo]. Puebla: Colegio de Postgraduados; 2011.

9. Skerman PJ, Cameron DG, Riveros F. Leguminosas forrajeras Tropicales. Roma: FAO; 1991.

10. Schiere JB. Backyard rabbit farming in the Tropics. $3^{\text {rd }}$ ed. Wageningen: Agrodok Publications; 1999.

11. Apraéz J.E. Comportamiento productivo del Cuy (Cavia porcellus) alimentado con pasto Kikuyo (Pennisetum clandestinum) obtenido bajo diferentes métodos de atención cultural. Pasto: Facultad de Ciencias Pecuarias, Universidad de Nariño; 2002.

12. Campling RC. 1970. Physical regulation of voluntary intake. In: Proceedings of the Third International Symposium (1969, Cambrige, GB). Physiology of Digestion and Metabolism in the Ruminant. Ed. A. Philipson. Cambridge: Newcastle upon Tyne; 1970. $226 \mathrm{p}$.

13. Keys J, Van Soest P, Young E. Effect of Increasing Dietary Cell Wall Content on the 
Digestibility of Hemicellulose and Cellulose in Swine and Rats. J Anim Sci. 1970; 31(6): 1172-1177.

14. FAO. Organización de las Naciones Unidas para la Alimentación y la Agricultura. 2016. A/RES/68/231. 